\title{
Im Delta des Poly-Werks - eine Annäherung
}

\begin{abstract}
„Luigi Nono äußerte einmal wie beiläufig $\mathrm{zu}$ seinem Streichquartett, daß diese Musik so erklingt und erklingen soll, wie er sie niedergeschrieben hat - daß sie aber auch eine ganz andere sein könnte ... Darin zeigt sich nicht nur der Gedanke des Fragmentalen einer einmal festgeschriebenen Gestalt, sondern ein grundsätzliches Infragestellen des Opus perfectum, verglichen mit den Ansätzen eines John Cage, aber in einer anderen utopischen Richtung."1
\end{abstract}

(Klaus Huber)

Während das Spektrum der Simultanaufführungen, wie bereits dargestellt, von losen und unverbindlichen Schichtungen verschiedener Werke im Sinne eines "Circus“ bis zu konkret inszenierten Verknüpfungen verschiedener Werke reicht und sich damit immer im Rahmen einer additiven Aggregation bewegt, sind die formalen Möglichkeiten komponierter Werkkopplungen vielfältiger gelagert. Wie auch die Simultanaufführungen sind komponierte Werksimultaneisierungen kein ausschließlich neues Phänomen des 2o. oder 21. Jahrhunderts, sondern lassen sich weiter zurückverfolgen. Der italienische Komponist Pietro Raimondi (1786-1853) schrieb bereits im 19. Jahrhundert groß angelegte Werke, die einzeln oder gleichzeitig aufgeführt werden konnten. ${ }^{2}$ Die 1948/49 von Darius Milhaud komponierten Streichquartette Nr. $14 \& 15$ sind wohl das erste Werkpaar, das sowohl eine singuläre als auch eine simultane Aufführung ausdrücklich vorsieht und damit zeitlich noch vor den Simultaneisierungen von John Cage datiert ist. In den letzten vierzig Jahren sind zahlreiche Kompositionen, beispielsweise von Klaus Huber, Adriana Hölszky, Chaya Czernowin, Julio Estrada oder Georg Friedrich Haas, entstanden, bei denen auf sehr unterschiedliche Weise die eigenen Werke der Komponisten entweder durch andere eigene Werke simultan kontextualisiert

1 Klaus Huber/Max Nyffeler (Hg.): Umgepflügte Zeit. Schriften und Gespräche (= Edition MusikTexte, Bd. 6), Köln: MusikTexte, 1999, S. 443.

2 Genauer: sechs Fugen, zwei Messen, drei Oratorien, zwei Opern. Auch im Finale des ersten Akts des Don Giovanni von Wolfgang Amadeus Mozart gibt es eine berühmte Tanzstelle, die drei verschiedene Tänze übereinanderlegt. 
oder umgekehrt geschlossene Werkeinheiten neu aufgespalten werden und so zu einer Koexistenz verschiedener Perspektiven gelangen. Damit umfasst die "Geschichte“ der Werksimultaneisierungen im 20. und 21. Jahrhundert mittlerweile einen Zeitrahmen von siebzig Jahren. Während das Aufbrechen der geschlossenen zur offenen Form von einem regen Diskurs begleitet war, wurde die Entwicklung des Poly-Werks nicht bewusst gefördert oder vorangetrieben. Sie fand abseits von Moden, Trends, Strömungen oder Debatten des Neue-Musik-Diskurses statt. Das sich zusammenfügende Gesamtbild ist das Resultat einer weitgehend unbewussten Entwicklung. Wenige Komponisten wie Claus-Steffen Mahnkopf oder Robert HP Platz thematisierten ihre Ansätze auch schriftlich, jedoch immer nur in Bezug auf das eigene künstlerische Werkverständnis. Aus diesem Grunde ist eine zusammenfassende theoretische und systematische Betrachtung überfällig.

\subsection{Die konzeptuelle Weiterentwicklung von Polyphonie und Werk im Poly-Werk}

Wie bereits in der Einleitung dargestellt, wurde der Begriff des „Poly-Werks“ 1990 von Claus-Steffen Mahnkopf in seiner Schrift „Kundgabe. Komplexismus und der Paradigmenwechsel in der Musik" eingeführt. ${ }^{3}$ Das Präfix "poly“ ist vor allem aus dem Kontext der Polyphonie vertraut. Während "poly“ erst einmal nur "mehr" meint, drückt der Begriffskontext „Polyphonie“ etwas über das Verhältnis der Dinge zueinander aus. Im Zusammenhang mit der Organisationsform des Kontrapunkts impliziert er eine simultane Verbindung selbstständiger, nach Unabhängigkeit strebender Linien und das sich daraus ergebende Spannungsverhältnis zwischen unabhängiger Linearität und vermittelnder Vertikalität.

Die Idee, ein Werk mit einem zweiten Werk zu synchronisieren, sodass ein drittes neues Werk entsteht, obwohl die Einzelwerke auch weiterhin unabhängig davon existieren, greift auch die alte polyphone oder kontrapunktische Idee, die eine lange Geschichte hat, auf faszinierende Weise wieder auf. Folglich ist auch der Begriff des Poly-Werks von der Vorstellung eines Weiterdenkens von Polyphonie auf formal-werkhafter Ebene geprägt. Mit der zunehmenden schriftlichen Auskomposition von Werksimultaneisierungen entstanden, dient er als Modell für die Übertragung divergierender Linienverläufe auf die Metaebene der Werkverläufe. Der Komponist Klaus Huber, in dessen Schaffen seit den 7oer Jahren auch Poly-Werke einen Platz haben,

3 Mahnkopf: „Kundgabe“. 
zieht eine direkte Verbindung zwischen Kontrapunkt und Formgestaltung und plädiert für einen erweiterten Begriff von Polyphonie: Eine „weitere Stufe von Kontrapunkt ist in diesem Sinne der formale Kontrapunkt, sozusagen ein FeldKontrapunkt oder Schichten-Kontrapunkt. Das alles kann Polyphonie sein.“4

Ein erweiterter Polyphoniebegriff erstreckt sich ebenso auf andere Anwendungen. Begriffe wie Polytonalität, Polymetrik, Polystilistik, Polyrhythmik oder Polytempo sind vertraute Kategorien, wenn es um Anordnungen geht, die den Zusammenfall des Verschiedenen ermöglichen. Claus-Steffen Mahnkopf entwickelte gar eine ganze Typologie von Polyismen, die Polymorphie, Polyprozessualität, aber auch Polyvektorialität und Polykonzeptualität ${ }^{5}$ umfasst und auf deren höchster Stufe die Kategorie des Poly-Werks als „Dissoziation des in sich geschlossenen und integralen Werks" ${ }^{\text {“6 }}$ steht. Der zugrunde liegende Leitgedanke entspricht der "Dissoziation musikalischer Diskursivität".7 Als rein satztechnische Kategorie ist Polyphonie für Mahnkopf die Dissoziation von Linien. Polymorphie definiert er als die Dissoziation von Gestalten, Polyprozessualität als die Dissoziation formaler Prozesse. Als Polyvektorialität beschreibt er die Dissoziation kompositorischer Techniken, und die Polykonzeptualität betrifft für ihn die Ebene der Semantik. Mahnkopf spricht von einem ideellen Splitten des Werks in mehrere Werke. Mündet die ideelle Splittung auch in eine materialisierte Werksplittung, ist der Fall eines Poly-Werks gegeben. ${ }^{8}$

Das Poly-Werk erscheint hier als letzte Steigerung eines kontrapunktischen Denkens, das sich in der Moderne mit den Worten Klaus Hubers als „Dialektik von materialen Möglichkeiten“9 ${ }^{9}$ zeigt. Das Prinzip „Note gegen Note“ wird zum „Werk gegen Werk“. Dazwischen existiert eine Skala polyistischer Varianten, die die verschiedenen Kategorien der Komposition einschließt und in vielen Fällen als Mittel bewusst eingesetzt wird, um die Stücke in ihrer Identität und Individualität einander noch markanter gegenüberzustellen. Die Relation dissonanter versus konsonanter Bewegungen findet ihren Ausdruck in der komplexen Wechselbeziehung synchroner und asynchroner Prozesse. Das schließt die Möglichkeit ein, die Verläufe der verschiedenen Parameter einer

4 Klaus Huber: Von Zeit zu Zeit. Das Gesamtschaffen. Gespräche mit Claus-Steffen Mahnkopf, Hofheim: Wolke, 2009, S. 155 .

5 Vgl. Mahnkopf: „Kundgabe“, S. 22.

6 Claus-Steffen Mahnkopf: „Theory of Polyphony“, in: ders. (Hg.): Polyphony \& Complexity (= New Music and Aesthetics in the 21st Century, Bd. 1), hg. von Claus-Steffen Mahnkopf, Hofheim: Wolke 2002, S. 38-53, hier: S. 49 .

7 Vgl. Mahnkopf: „Kundgabe“, S. 21 oder auch Kritik der Neuen Musik, S. 118.

8 Vgl. Mahnkopf: „Theory of Polyphony“, S. 47-5o.

9 Huber: Von Zeit zu Zeit, S. 156. 
Komposition auch gegeneinanderzusetzen, sie aneinander brechen zu lassen, sie zu enthierarchisieren, um jegliches gemeinsames Streben nach einer Synthese und einer vereinheitlichten Syntax auszuhebeln. ${ }^{10}$ Dieser Ansatz integriert Verfahren, die vor allem das Nebeneinander, das Differente, die Unabhängigkeit der Ereignisse ermöglichen und betonen. Zum ästhetischen Ideal der Polyphonie in der europäischen Tradition verhält er sich allerdings konträr, da dort der Entwurf der einzelnen Stimmen immer im Hinblick auf die Bildung einer Einheit gerichtet war. Musikalische Ereignisse und Entwicklungen wurden in einer mehrstimmigen Anlage so geplant und verknüpft, dass sie in der vollkommenen Synthese aufgingen. Der Kontext des Poly-Werks dagegen ermöglicht es ebenso, Kompositionen zu schaffen, die in ihrem Zusammengang in eine gebrochene Synthese münden. Ein derart erweitertes polyphones Denken fungiert hier in erster Linie als gemeinsamer Rahmen, der die Wahrnehmung des Verschiedenen ermöglicht und in unterschiedlichen Abstufungen eine formale, materielle und organisatorische Verbindlichkeit erzeugt - im Gegensatz zur Schichtung als pluralistischem Übereinander heterogener Stücke und Materialien ohne intendiertes Konnektiv. Wie bei einem rein polyphonen Hörerlebnis (z. B. einer Fuge) oszilliert der Hörer in unterschiedlichen Graden und je nach Exposition der Stimmen zwischen dem selektiven Verfolgen des einzelnen Werkverlaufs und dem Gesamteindruck.

Die gebrochene Synthese im Delta des Poly-Werks ist eine Möglichkeit, das ursprünglich polyphone Miteinander um ein Neben- und ein Gegeneinander der Einzelstränge zu erweitern. Gegenüber dem historischen Modell der Polyphonie erweitert sich der Polyphoniegedanke im Poly-Werk noch um eine weitere Richtung. Denn das „Poly“ muss gar nicht mehr nur einseitig als eine Ver,mehr"ung zu verstehen sein, sondern kann umgekehrt auch eine Abspaltung, eine Dissoziation, evozieren. So ist Mahnkopfs theoretische Konzeption des Poly-Werks ausschließlich auf die „Dissoziation“ des Werkgedankens ausgerichtet. Diese subtraktive Möglichkeit markiert eine weitere qualitative Unterscheidung zur Simultanaufführung, die prinzipiell immer additiv ist. Beide Formen, die gebrochene Synthese und die Desynthese, gehen auf ein entscheidendes Kriterium in der Werkgenese eines Poly-Werks zurück: das selbstständige und autonome Dasein der Einzelstränge. Während beispielsweise in einer Fuge die verschiedenen Stimmen in ihrer Beweglichkeit gegenüber einem homophonen Satz lediglich eine gewisse Eigenständigkeit

10 Aus Mahnkopfs Sicht erwächst diese Möglichkeit direkt aus dem mit der Auflösung der Tonalität einhergehenden Wegbrechen formstiftender Syntax. Zwölftontechnik und Serialismus kritisiert er als unzulängliche Versuche, „über vereinheitlichtes Material Einheit zu stiften“. Vgl. hierzu Mahnkopf: Kritik der neuen Musik, S. 111. 
innehaben, ist diese Eigenständigkeit mitnichten so stark, dass sich die einzelnen Stimmen auch außerhalb des Kontextes Fuge aufführen ließen. Die Stimmen haben hier den Status von Modulen, die ineinandergreifen und einander auch in ihrer Hervorbringung bedingen, sich aber nicht voneinander lösen können. Dagegen ist im Poly-Werk die selbstständige Aufführbarkeit einzelner Teile eine unhintergehbare Grundbedingung. Die der Polyphonie immanente Spannung zwischen Horizontalität und Vertikalität erstreckt und verlagert sich damit auch auf eine Spannung zwischen dem „Einen“ und dem "Mehr", zwischen "Werk" und „Poly“. Während es sich bei einem polyphonen Werk immer nur um eine einzige Werkidentität handelt, setzt sich das PolyWerk aus mehreren Werkidentitäten zusammen. In den Vordergrund rückt nun die Qualität ihres wechselseitigen Verhältnisses, ihre Lagebeziehung im Raum und in der Zeit. Vor diesem Hintergrund sind Kategorien wie Polyphonie und Kontrapunkt nicht mehr nur rein satz- oder kompositionstechnische Organisationsformen. Sie entfalten auch eine konzeptionelle Dimension, die die Frage nach der Identität im Verschiedenen stellt und vor allem auf diese Weise über den offenen und geschlossenen Werkbegriff hinausweist und einen dritten Weg einschlägt.

„[D]as im Erscheinenden noch nicht Erschöpfte, im Manifestierten vermittelte, aber nicht selber ganz Manifestierte - ist Identität."11

(Ernst Bloch)

Der erweiterte Polyphoniegedanke im Poly-Werk überführt die ursprüngliche Selbstständigkeit einzelner Stimmen auf eine neue Stufe einer tatsächlichen und realen Verselbstständigung. Dieser Schritt hat wesentliche Folgen für die Werkkonzeption. Er führt dazu, dass das „Werk“ nicht mehr als eine deutlich abgeschlossene und vor allem unteilbare Einheit betrachtet werden kann, sondern dass es verschiedene, aber trotzdem weiterhin in sich geschlossene Teilwerke in sich integriert, dass es also damit auch "teilbar" wird. Damit entzieht sich der Werkbegriff seiner Eindeutigkeit, denn es stellt sich die Frage: Was ist jetzt das Werk? Weder ist es eindeutig das Ganze, das alles umfasst, noch ist es nur das geteilte Werk, das ebenso für sich selbst stehen kann. Jede

11 Ernst Bloch, Subjekt-Objekt. Erläuterungen zu Hegel, Frankfurt am Main: Suhrkamp, 1962, S. 510 . 
Werkversion zeigt nur eine mögliche Seite und bleibt damit immer nur ein Ausschnitt des Ganzen.

Ein Seitenblick auf den Identitätsbegriff in den Sozialwissenschaften macht eine interessante Analogie sichtbar. Die Vorstellung vom menschlichen Subjekt als einer singulären, in sich selbst geschlossenen zentrischen Einheit mit einer eigenen unteilbaren Identität ist dort längst korrigiert worden. Ab Mitte des 20. Jahrhunderts wurde Identitätsbildung zunehmend vor dem Hintergrund eines komplexen und interaktiven Werdensprozesses diskutiert. ${ }^{12}$ Im Zuge der "dialogischen Wende“ galt das Individuum zunehmend nicht mehr als unteilbare Einheit. Vielmehr setzte sich die Vorstellung einer Zusammensetzung verschiedener Teilidentitäten durch, die sich auf der Grundlage intersubjektiver Prozesse entwickeln und deren verschieden gelagerte Anforderungen in ein individuelles Gleichgewicht gebracht werden müssen. Die Entwicklung von Identität bindet sich damit an ein Gefüge von Beziehungen, die außerhalb der Identität selbst liegen. Ebenso entspricht das Werkkonzept im Poly-Werk nicht mehr nur einer einzelnen individuellen oder originellen Werkidentität. Die Zusammensetzung verschiedener Teilwerke und damit auch verschiedener Teilidentitäten bedingt folglich entweder die Öffnung der Werke hin zu einer Beziehung oder aber die Hervorbringung der Teilwerke aus einer konkreten Beziehung.

„D'un point de vue anthropologique, l'identité est un rapport et non pas une qualification individuelle comme l'entend le langage commun. Ainsi, la question de l'identité est non pas ,que suis-je?', mais ,qui je suis par rapport aux autres, que sont les autres par rapport à moi?' Le concept d'identité ne peut pas se séparer du concept d'altérité." ${ }^{13}$

12 Aus der Überfülle der Literatur siehe hierzu beispielsweise Heiner Keupp u. a. (Hg.): Identitätskonstruktionen. Das Patchwork der Identitäten in der Spätmoderne, Hamburg: Rowohlt, 2002; Heiner Keupp/Renate Höfer (Hg.): Identitätsarbeit heute. Klassische und aktuelle Perspektiven der Identitätsforschung, Frankfurt am Main: Suhrkamp, 1998. Es würde den Rahmen dieser Arbeit sprengen, diese Debatte in der Fülle ihrer Stimmen hier angemessen aufzugreifen. Der Verweis auf die Diskussion in den Sozialwissenschaften ist lediglich als Anregung zu verstehen, das geschlossene Werkkonzept zu erweitern, ohne es negieren zu müssen. Analogien zwischen den beiden Sphären wären für musikwissenschaftliche oder transdisziplinäre Forschungen zum Werkkonzept sicher produktiv.

13 Äußerung von Jean-Francois Gossiaux im Juni 1997 auf einer Konferenz der DIF POP (Entreprise de diffusion des revues scientifiques) über „L'identité nationale“, zitiert nach Jean-Claude Ruano-Borbalan, L'identité. L'individu, le groupe, la societé, Paris: Sciences Humaines Éditions, 1998, S. 2. Übersetzung des Verf.: „Aus anthropologischer Sicht ist Identität eine Beziehung und keine individuelle Eigenschaft, wie sie in der Umgangssprache verstanden wird. Die Frage nach der Identität lautet also nicht: ,Wer bin ich?‘, sondern: ,Wer bin ich im Verhältnis zu anderen, wer sind die anderen im Verhältnis zu mir?' Das Konzept der Identität kann nicht vom Konzept der Alterität getrennt werden.“ 
Diese Verschiebung von einem einheitlich gedachten und abgrenzbaren Subjekt hin zu einer Konstellation von Identitäten ist vor dem Hintergrund eines gesellschaftlichen Transformationsprozesses und des gesteigerten Anspruchs an Identitätsbildung ein interessantes Modell in Bezug auf die Situation der Form und der Werkidentität im Poly-Werk. Hier wird der musikalische Text aus der Spannung zwischen einer Einheit und einer Vielfalt verschiedener Funktions- und Rollenanforderungen heraus entwickelt. Selbst wenn der traditionelle Werkbegriff im Konzept des Poly-Werks eingeschlossen ist, geht der Schritt vom Mono- zum Poly-Werk mit einer Modifikation, wenn nicht gar einer Umkehrung etablierter Normen und Maßstäbe im Hinblick auf den Werkbegriff einher. Denn ebenso wie Harmonik, Rhythmik und Klangfarblichkeit im 20. Jahrhundert eine Ausdifferenzierung erfahren haben, liegt im Falle von Poly-Werken eine Ausdifferenzierung von Form, Werk und Werkidentität vor. Statt auf die Zuschreibung einer zentrisch geschlossenen und vollendeten Werkidentität gerichtet zu sein, konstituiert sich "Werk“ nur noch im Plural. Die intraformale Gestaltung des musikalischen Textes ist nur noch ein Teilaspekt und als solcher nicht mehr maßgebend für die Komposition des Ganzen. Die (Teil-)Werke entstehen nunmehr in einer ein- oder wechselseitigen Abhängigkeit. Die intraformale Entwicklung und Wirkung ist sowohl für sich als auch in Bezug auf ein Formgeschehen zu sehen, das sich außerhalb der werkeigenen Entwicklung vollzieht und ein interformales Verhältnis, ein Geflecht der Wechselwirkungen hervorbringt. Maßstab ist nun nicht mehr die Individualität eines Werks, sondern die Vereinbarkeit von Singularität und Individualität einerseits und Wandlungsfähigkeit und Adaptivität andererseits und damit auch die Korrelation zwischen Intra- und Interform. Das Konzept von Werkidentität im Poly-Werk ist also immer an das Konzept einer anderen Identität gebunden, die ebenso für sich stehen und existieren kann. „Die Innenbeziehung, das Selbstverhältnis und die Beziehung zum anderen bilden die beiden ineinander verschlungenen und einander bedingenden Bestandteile von Identität."14

In dieser chamäleonartigen Konstellation wird das Werk zur Schnittstelle verschiedener Identitäten. Jedes Werk ist als Teilwerk auch ein Erfahrungsraum für ein anderes Teilwerk. Je nach Kontext wird die Wirkungsdimension eines musikalischen Textes dadurch entweder gesteigert, gefördert oder umgekehrt auch unterdrückt, entstehen Spannungen zwischen einer forminneren Logik und einer kollektiven Metaform. Krappmanns Metapher der

14 Sigrun Anselm, „Identifizierung und Selbstbehauptung. Überlegungen zu einer aktuellen Dimension des Anerkennungskonflikts“, in: Keupp/Höfer (Hg.): Identitätsarbeit heute,

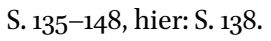


„balancierende[n] Identität ${ }^{415}$, die trotz der divergierenden Ansprüche, Widersprüche und Reibungen gefordert ist, eine spezifische Form der Kohärenz zu finden, drückt diese Dichotomie sinnfällig aus.

\subsection{Künstlerisches Gestalten in der Differenz}

Im Gegensatz zum alleinstehenden, absoluten Werk zeichnet sich das PolyWerk durch die Option einer simultanen Verkopplung innerhalb einer Werkgruppe aus. Die eingangs genannten Kriterien der Ergänzbarkeit verschiedener in sich geschlossener Werke und der Originalübertragung des Notentextes in die verschiedenen Kontexte sehen grundsätzlich keine Möglichkeit der Überarbeitung und Adaption des primären Notentextes für die verschiedenen Konstellationen vor. Die Freiheit, den Notentext für den neuen Zusammenhang noch einmal zu ändern, ihn zu überdenken und in seine Struktur einzugreifen, entfällt beim Poly-Werk. Aus künstlerischer Sicht stellen die Bewahrung des Notentextes versus seine Überarbeitung zwei unterschiedliche Herangehensweisen im kompositorischen Prozess dar. ${ }^{16}$ Hier liegt die entscheidende Weggabelung zwischen der Komposition eines einfachen Werks und der eines Poly-Werks. Werke innerhalb der Werkgruppe sollen in sich schlüssig, untereinander anschlussfähig und trotzdem verschiedenartig sein. Das absolut zu denkende Werk muss im gleichen Moment offen für eine Vernetzung oder eine Abspaltung sein. Die von Darius Milhaud komponierten Streichquartette Nr. 14 und 15, die sich zum Oktett zusammenschließen können, sind in diesem Zusammenhang musikgeschichtlich ein erster Meilenstein (vgl. Kapitel 4.3.1). Beide Stücke sind explizit auf den Zusammengang konzipiert und vom Verlag auch als Simultanpartitur herausgegeben worden. Die Gestalt beider Streichquartette ist festgeschrieben, der Notentext ändert sich in der simultanen Version nicht, wirkt sich aber natürlich ganz anders aus. Es ist bemerkenswert, dass es sich bei diesem frühen Beispiel bereits um eine heterogen angelegte Konstellation handelt. Beide Quartette teilen zwar die gleiche Besetzung, die

\footnotetext{
15 Siehe beispielsweise Lothar Krappmann, „Die Identitätsproblematik nach Erikson aus einer interaktionistischen Sicht“, in: Keupp/Höfer (Hg.): Identitätsarbeit heute, S. 66-92, hier: S. 81.

16 Beispielsweise integriert das Orchesterstück Masse, Macht und Individuum von Vinko Globokar vier Solostücke als Dialoge über Wasser, Erde, Feuer und Luft für Percussion, Kontrabass, elektrische Gitarre und Akkordeon in die Partitur. Zwar bleiben die Soli als solche klar erkennbar und behalten auch ihren solistischen Status und ihre Identität. Jedoch weichen die Fassungen der Einzelsoli und Orchestersoli voneinander ab, denn Globokar hat Abschnitte vertauscht, variiert, ergänzt oder auch ganz weggelassen.
} 
gleiche Dauer, das gleiche Metrum und Tempo. Trotzdem exponiert Milhaud in den Quartetten sehr unterschiedliche Charaktere. So ist das erste Quartett des ersten Satzes vor allem melodisch-linear, das zweite Quartett eher scherzoartig. Trotz des unterschiedlichen Charakters gibt es kleine Brücken im Tonsatz. So werden in beiden Quartetten die Themen durch die ersten Geigen gespielt. Beide Themen beginnen mit demselben Anfangston a, der Dreiklangston sowohl in F-Dur als auch in D-Dur ist, den tonalen Durzentren beider Quartette. Der Synkopenrhythmus beider Quartette verbindet sich im zweiten Takt zu einem Rhythmus und harmonischen Block. Die chromatische Linie der Bratsche aus dem Quartett Nr. 14 (vgl. Kapitel 4.3.1) hat damit je nach Kontext eine andere Funktion. Kompositionstechnisch ist hier vor allem interessant, wie Milhaud die Vergleichzeitigung zweier Werke auf der Basis seiner polytonalen Kompositionsverfahren heraus entwickelt. Vor diesem Hintergrund erscheint das Stück nicht als isoliertes, singuläres Experiment in seinem Schaffen, sondern als natürliche Konsequenz eines polytonalen und polylinearen Denkens und Experimentierens, das auch bei Milhaud - nach seiner eigenen Aussage - seine Wurzeln in der bewussten Wahrnehmung des Verschiedenen hat. Er selbst spricht von Kindheitserinnerungen, von der Wahrnehmung verschiedener Klänge und Geräusche des Straßentreibens vor seinem Haus. ${ }^{17}$

Milhaud hat seine Werke in einer Simultanpartitur komponiert. Bei der Niederschrift des einen Quartetts musste er die Gegenwart der anderen beiden Versionen mitdenken und seine Entscheidungen von Anfang an in Bezug auf die verschiedenen Kontexte treffen. Dieses Vorgehen markiert einen qualitativen Umschlag im kompositorischen Prozess. Ich nenne diesen Prozess frei nach Wolfgang Welsch, der in Bezug auf die Praktiken der Postmoderne vom „Operieren inmitten der Differenz" gesprochen hat, ${ }^{18}$ ein „künstlerisches Gestalten in der Differenz". Meiner Meinung nach veranschaulicht diese Denkfigur die Besonderheit und das Wesen dieser Praxis sinnfällig. Denn sie verweist auf die gespaltene Werkintention, die Stücke als Einzelne auszuarbeiten und gleichzeitig ihr Verhältnis zueinander zu komponieren. Dadurch erweitert sich der Interpretationsraum eines Werks bereits in der Phase der Komposition. In dem Moment, in dem ein Komponist ein Werk, Werkteil oder einzelne Stimmen in andere formale Zusammenhänge stellt, legt er sich nicht auf einen Blickwinkel fest, sondern entscheidet sich dafür, das Material unterschiedlich interpretieren, umfunktionieren und in einen diskursiven Verlauf stellen zu

17 Darius Milhaud, Notes sans musique, Paris: Juillard, 1949, S. $16 f$.

18 Wolfgang Welsch: „Einleitung“, in: Wege aus der Moderne. Schlüsseltexte der PostmoderneDiskussion, hg. von Wolfgang Welsch, Weinheim: VCH, 1988, S. 1-43, hier: S. 40. 
können. So gibt beispielsweise ein Solostück, das sich mit anderen Stücken zu einem kammermusikalischen Werk verbinden kann, in der simultanen Version seinen solistischen Status auf, erlebt einen Funktionswandel und eine Neuinterpretation seines Textes durch denselben Komponisten. Das Möglichkeitsfeld an festgeschriebenen gültigen Werkversionen, die variable Funktionalität eines Werkes in verschiedenen Zusammenhängen, erweitert auch die interpretatorischen Möglichkeiten der Interpreten und Rezipienten und macht eine flexible und dynamische Haltung zum musikalischen Text erforderlich.

Die Janusköpfigkeit der Werke ist gleichermaßen auch eine Janusköpfigkeit der künstlerischen Praxis, speziell des kompositorischen Prozesses. In diesem bedeutet sie nicht einfach nur Öffnung. Auf der einen Seite werden zwar neue Möglichkeiten der Materialentwicklung geschaffen, auf der anderen Seite aber ebenso limitiert und begrenzt. Jedes Einzelwerk stellt Bedingungen auf, die das andere Werk in seiner Entfaltung einschränken. Ein einmal gesetzter Parameter beeinflusst auch die Parametersetzung im anderen Stück und führt zu einer wechselseitigen Determination der endgültigen Form.

Eine wichtige Rolle für die Gestaltung des Verhältnisses der Werke untereinander spielt dabei bereits die Herangehensweise im Kompositionsprozess, in dem die Stücke entweder sukzessive oder simultan komponiert werden. Bei einer sukzessiven Kompositionsweise ist das erste Stück in seiner Entstehung unabhängiger als das zweite, das "gezwungen“ wird zu reagieren. Valerio Sannicandro beispielsweise hat seine beiden Orchesterwerke Windströme und Seelenströme nacheinander komponiert. ${ }^{19}$ Auch Chaya Czernowin komponierte die Werke des Anea Crystal Cycle nacheinander, legte aber das erste Stücke Seed I für die Komposition des zweiten Stückes bewusst zur Seite. Die zweite Möglichkeit der Werkgenese ist die simultane Komposition der Werke. Hier wird es meistens so sein, dass am Anfang ein übergreifender Plan entworfen wird, der zur Orientierung dient und festhält, inwieweit die Stücke aufeinander reagieren und welche Funktionen sie in Bezug aufeinander haben. Mithilfe dieses Plans können dann die einzelnen Stücke wiederum auch sukzessive auskomponiert werden, eine Mischung aus beiden Vorgehensweisen.

19 Das Orchesterstück Windströme ist eine differenzierte Orchestration tonloser Luftgeräusche. Es entstand zuerst, erlebte vor der Komposition des zweiten Stückes seine Uraufführung und gab dem Orchesterstück Seelenströme, das im Gegensatz zum ersten Stück eine Entfaltung klanglichen und melodischen Materials ist, mehrere Bedingungen wie die Dauer der Formabschnitte und die Metrik vor. 


\subsection{Die Erweiterung des Begriffsfeldes}

Der Begriff des Poly-Werks wird von den meisten Komponisten nicht verwendet oder sogar abgelehnt. Der Grund ist vermutlich, dass er eine Anknüpfung an traditionelle ästhetische Konzepte suggeriert und ihm im Kern die Objekt- und Werkhaftigkeit eingeschrieben ist. Tendenziell wird eher auf Beschreibungsformen zurückgegriffen, die das spezifisch Prozesshafte der jeweiligen Zusammenführung herausstellen. Lediglich Ulrich Kreppein bezeichnet seine Echoräume tatsächlich als „Polywerk“. ${ }^{20}$ Claus-Steffen Mahnkopf schrieb über das Konzept des Poly-Werks: „Dieses Formprinzip ist äußerst wandlungsfähig und offen für eine Unzahl von Kombinationen“21. Das spricht auch erst einmal für eine Vielfalt an Beschreibungsformen und Begriffen. Chaya Czernowin beschreibt ihren Zyklus Anea Crystal Cycle einfach mit der Möglichkeit einer simultanen Aufführbarkeit. ${ }^{22}$ Adriana Hölszky untertitelt ihre Hängebrücken mit „Doppelquartett an Schubert“23. Juliane Klein bezeichnet den Zyklus Aus der Wand die Rinne als einfache „Werkgruppe“24. Bernd Franke spricht von einem „komplexen Vernetzungsprinzip“25 in Bezug auf seinen Solo-xfachZyklus, Aaron Cassidy beschreibt seine Stücke als „conglomeration of three interconnected, simultaneous trios for seven players“26, Georg Friedrich Haas nennt „... Einklang freier Wesen ..." ein „interlacing of ten completely, independent autonomous solos, smaller sub-ensembles“, und Simon SteenAndersen beschreibt Next To Beside Besides als „choreographical translations“27. Robert HP Platz verwendet an verschiedenen Stellen den metaphorischen Begriff der „Raumpolyphonie“ oder spricht auch von einer „Polyphonie verschiedener Formen“28.

Bis auf Ulrich Kreppein vermeiden alle Komponisten einen Werkbegriff, obschon die Möglichkeit der Simultaneisierung im kompositorischen Prozess

$20 \quad$ Ulrich Kreppein, Echoräume für vier Ensembles, Partitur, 2016/17, Berlin: Edition Juliane Klein.

21 Mahnkopf: Kritik der neuen Musik, S. 118.

22 Chaya Czernowin, Anea Crystal Cycle, Partitur, 2009, Mainz: Schott.

23 Adriana Hölszky, Hängebrücken. Streichquartette/Doppelquartett an Schubert, Wiesbaden: Breitkopf \& Härtel, 1991.

24 Juliane Klein, Aus der Wand die Rinne 1-14, 1996-2014, Berlin: Edition Juliane Klein.

25 Vgl. http://berndfranke.de/werke-2/solo-xfach/ensemble/ (letzter Aufruf am 14.3.2020).

26 Vgl. http://aaroncassidy.com/product/the-green-is-either/ (letzter Aufruf am 14.3.2020).

27 Vgl. Simon Steen-Andersen, Next To Beside Besides 0-11, 2003-2007, Frederiksberg: Edition S.

28 Vgl. https://www.rhpp.de/index.cfm?id=3664 (letzter Aufruf am 14.3.2020); siehe auch Richard Toop: „Immer weiter. ,Formale Polyphonie“ und Robert HP Platz' Donaueschinger Zyklus“, in: Musik \& Ästhetik, Heft 6, 1998, S. 61-76. 
fast immer auf die einzelne Individualstruktur der Werke zurückwirkt und teilweise auch Simultanpartituren existieren. ${ }^{29}$ Die meisten Umschreibungen und Begriffe verweisen etwas unbestimmt auf die von vornherein intendierte qualitative Werkverknüpfung. Die eigentliche Besonderheit, nämlich die gleichzeitige Aufführbarkeit bei gleichzeitiger Autonomie der Einzelwerke ist dagegen nicht immer auf den ersten Blick greifbar. Aufgrund der unklaren Begriffslage im Umfeld des Poly-Werks soll im Folgenden ein Überblick über die Begriffsmöglichkeiten und ihre Einordnung versucht werden.

Die "Simultanaufführung“ ist eine simultane Zusammenführung präexistenter Werke durch Interpreten und damit eindeutig eine Aufführungspraxis, die verschiedene Werke im Nachhinein additiv zusammenführt. Die Zusammenführung kann das Resultat analytisch bestimmter Verbindungslinien der musikalischen Texte sein. Sie kann aber ebenso auch lose und unverbindlich sein, wie bei einem "Circus“. Ein "Circus" stellt zudem den Wert der Quantität der zu schichtenden Stücke über den Wert der Qualität der Verbindung. Das begriffliche Äquivalent "Simultanwerk“ wird bis zu diesem Zeitpunkt wohl noch nicht verwendet. Der Begriff würde auf die parallele Zusammenführung als kompositorische Praxis verweisen und ebenso einen additiven Zusammenschluss von Werken implizieren. Dagegen kann das „PolyWerk" neben den additiven auch subtraktive Verfahren, innere qualitative Aufspaltungen von Werkentitäten, Dissoziationen und Externalisationen beinhalten. Bildlich gesprochen handelt es sich bei den subtraktiven Vorgängen um eine Umkehrung oder Negation des additiven Prinzips und damit bereits um eine Variation des Polyphonieprinzips.

Der entscheidende Unterschied zur Simultanaufführung besteht darin, dass im Poly-Werk die Form der Verbindung vom Komponisten von vornherein antizipiert wird. Das hat Folgen für den Charakter und den Aufbau der Einzelwerke und die Gesamtdisposition, die oftmals eine formale Herausforderung darstellen und genaue präkompositorische Planungen und Überlegungen bezüglich der Anschlussstellen und der Synchronisierung erforderlich machen. Der Begriff des Poly-Werks wird bei Mahnkopf in Verbindung mit einem polyphonen Denken verwendet und zielt auch hier auf das qualitative Moment der Verknüpfung. Aus diesem Grund ist er nicht für alle Werkkopplungen der passende Begriff. Vermutlich wird er auch deswegen von

29 Die Zyklen Anea Crystal Cycle (Chaya Czernowin), Hängebrücken (Adriana Hölszky), Medusa (Claus-Steffen Mahnkopf) oder auch „... Einklang freier Wesen ...“ (Georg Friedrich Haas) existieren als Simultanpartituren. In anderen Fällen sind die Werke einzeln oder im sukzessiven Verbund erhältlich. Ist ein Poly-Werk als Simultanpartitur werkhaft fixiert, kann es als solches gehandelt, gelesen und studiert werden. 
den meisten Komponisten gemieden, weil er in den Schriften von Mahnkopf Teil eines Denkmodells ist, das spezifische Ansprüche an die Werkkonzeption und die Werkrezeption formuliert, und bei ihm im Zusammenhang mit dem Komplexismus gebraucht wird..$^{30}$

Claus-Steffen Mahnkopf geht in seiner Definition des Poly-Werks - die in erster Linie aber für seine eigenen Werke Gültigkeit hat und in vielen Punkten nicht generalisierbar ist - von der Dissoziation, der Spaltung einer Einheit, statt einer Addition aus:

\begin{abstract}
„The poly-work, finally, is the dissociation of the work as an organically defined whole: within a consistently integrally and organically composed work are located independent, in themselves equally organically composed works which, like organelles (cellular sub-components), make indispensable contributions to the overall organization and can, in their ex-corporated form, be played as autonomous works. The very attraction of the poly-work lies in the formal strategy of distributing the internal components with differing (but shorter) durations than the composite structure, in order thus to effect an internal dissociation of the temporal organization without destroying it. ${ }^{431}$
\end{abstract}

Mahnkopfs Entwurf einer integrativ-verschachtelten „Reinform“ des PolyWerks beschreibt vor allem den Aufbau seiner eigenen Poly-Werke. Bereits die Integration von kleineren Subwerken ist auf viele andere Fälle nicht mehr übertragbar und damit auch nicht die Definition als solche. Für eine allgemeine Verwendung des Begriffs ist meines Erachtens die Antizipation einer qualitativen Verknüpfbarkeit im kompositorischen Prozess unter Einschluss sowohl additiver als auch subtraktiver Verfahren ausschlaggebend.

Der Begriff „Werkschichten“ wird von Andreas Holzer in seinem Buch Zur Kategorie der Form in Neuer Musik $k^{32}$ synonym zum Begriff des Poly-Werks verwendet. Die Begriffe werden nicht voneinander abgegrenzt. Der Begriff Werkschichten impliziert zwar die zentrale Bedeutung des Simultanen, ist aber insofern missverständlich, als es sich auch um einzelne Schichten eines Werks handeln könnte. Außerdem wird das Verhältnis der Einzelwerke zueinander auf diese Weise von vornherein auf eine Schichtung, also eine einfache Überlagerung reduziert und nicht auf das konstruktive und verknüpfende interformale Moment Bezug genommen.

Der Begriff „Formpolyphonie“ wurde im Zusammenhang mit den Werken von Robert HP Platz geprägt, dessen Schaffen vielfach von Werksimultaneisierungen durchzogen ist. Das Phänomen der Werküberlagerung nimmt

3 Vgl. z. B. Mahnkopf: Kritik der neuen Musik, S. $112 \mathrm{ff}$.

31 Mahnkopf: „Theory of Polyphony“, S. 49.

32 Holzer: Zur Kategorie der Form in neuer Musik, S. 506-510. 
einen zentralen Platz in seinem Gesamtwerk ein. Viele seiner Werke sind untereinander anschlussfähig, überlappen sich oder können parallel aufgeführt werden. Beispiele sind:

- up $(+)$ down $(+)$ strange $(+)$ charme

- Wunderblock

- dur (+) moll (+) hören

- Branenwelten

- Unter Segel (+) Boutaden (+) Hülle 2

- Horizont $(+)$ Architektur

- Hülle 1

- Grenzgänge (+) STEINE

- Flötenstücke für Altflöte und Ensemble etc. ${ }^{33}$

Der Terminus „Formpolyphonie“ streicht den Aspekt der Form heraus und ist eher geeignet, die Divergenz von Formen, Formtypen und musikalischen Abläufen innerhalb einer Werkentität zu bezeichnen. Diese ist nicht auf das Poly-Werk beschränkt. Die Schichtung und Fusion verschiedener Formtypen innerhalb einer Form wurde weit früher praktiziert, insbesondere in einigen Werken der Zweiten Wiener Schule. ${ }^{34}$ Da es nun aber auch Poly-Werke gibt, die gerade in ihrer Formhaftigkeit einheitlich sind und ihre Divergenzen auf anderen Ebenen austragen, ist dieser Begriff auch bei Robert HP Platz als übergeordnete Kategorie eher missverständlich. Hier scheint der - bis jetzt wohl auch noch nicht verwendete - Begriff der "Werkpolyphonie“ besser geeignet zu sein, da er sich nicht nur auf ein formales Spannungsverhältnis verengt. Im Gegensatz zum „Poly-Werk“ zielt er außerdem weniger auf einen Abschluss, sondern deutet auf ein offenes Metaprojekt im Gesamtschaffen hin, wie es bei Platz auch tatsächlich der Fall ist.

Neben den bereits aufgezählten Varianten können weitere Begriffe nützlich sein, gerade wenn sie assoziativ auf den Charakter der Kopplung verweisen. Der Begriff „Werkpaar" ist bei der Konstellation zweier gleichstarker Werkeinheiten zutreffend, beispielsweise im Fall eines verdoppelten Streichquartetts. Der Begriff „Werkcluster“ ist treffend, wenn es um ähnliche und einander nahe Solostücke geht, die sich zu Ensemblestücken kombinieren lassen. Ich verwende ihn für die Werkzyklen von Julio Estrada, Simon SteenAndersen und Juliane Klein. Als „Subwerke“35 bezeichnet Claus-Steffen

33 Siehe auch https://www.rhpp.de/index.cfm?id=3251 (letzter Aufruf am 14.3.2020).

34 Siehe hier zum Beispiel Theodor W. Adorno in seinem Vortrag über die „Form in der Neuen Musik" in Thomas (Hg.): Form in der Neuen Musik, S. 18 f.

35 Claus-Steffen Mahnkopf: „Medusa. Concerning Conception, Poetics and Technique“, in: ders. (Hg.): Polyphony \& Complexity, S. 245-265, hier: S. 247. 
Mahnkopf die kleineren Kammermusikkompositionen als Teilkompositionen seines Zyklus Medusa. Das übergreifende Orchesterstück kann wiederum als „Dachkomposition“ oder „umbrella composition“36 bezeichnet werden. Klaas Coulembier plädiert in seiner Dissertation außerdem sinnvollerweise für eine präzise Unterscheidung zwischen autonomen und unabhängigen Werken. Die Einzelwerke im Poly-Werk sind zwar autonom, aber nicht vollständig unabhängig voneinander, da sie ja in Bezug aufeinander, entstanden sind. ${ }^{37}$

Berücksichtigt man die verschiedenen Aspekte, scheint der Terminus „Poly-Werk" - trotz der spürbaren Ablehnung von Seiten der Komponisten als Oberbegriff das Phänomen meines Erachtens doch am genauesten zu fassen. Der Bezug zum Konzept der Polyphonie verweist auf das spannungsvolle Kräfteverhältnis zwischen einer sich verselbstständigenden Sukzessivität und einer verbindenden Simultaneität. Der Bezug zum Kontext des Werks bringt durch die Nebeneinanderstellung der Wortkomponenten "Poly“ und „Werk“ die Spannung zwischen der ausgedrückten Mehrzahl und dem als Einzahl gedachten Gebilde des "Werks“ aus. Damit erweitert der Begriff sowohl das Werkkonzept als auch das Konzept von Polyphonie und stellt so eine konstruktive Alternative zur Negation des Werkbegriffs dar. Denn ein Werkbegriff, der entweder nur zur Eindeutigkeit oder zur Negation verdammt ist, ist keiner Weiterentwicklung fähig.

Die folgende Liste umfasst Werke, auf die die Kriterien des Poly-Werks zutreffen. Auch diese Liste erhebt keinen Anspruch auf Vollständigkeit. Da sich der Begriff „Poly-Werk“, für den ich als Oberbegriff plädiere, für die Neue Musik erst langsam durchsetzt, ist es aufgrund der Diversität der Bezeichnungen und mitunter auch der Nichtbezeichnung bisweilen schwierig, Poly-Werke „ausfindig“ zu machen. Zudem gibt es eine Reihe von Werken, die sich auf den ersten Blick sehr stark an das Konzept annähern, es dann aber doch nicht in derselben Konsequenz realisieren, beispielsweise indem einzelne Module, wenn sie unabhängig von der simultanen Version gespielt werden, noch einmal überarbeitet werden. Die Unterscheidung ist nicht herabsetzend gemeint, aber es ist einfach etwas anderes. Die folgenden Werke sind mehrheitlich auch nicht explizit als Poly-Werke konzipiert worden. Was sie vereint, sind die gleichartigen Bedingungen, die sich die Komponisten bei der Komposition als Aufgabe gestellt haben, das heißt die Möglichkeit einer Neukontextualisierung und Vergleichzeitigung eines autonomen Werks durch eine simultane Aufführung in oder mit mindestens einem anderen Werk oder umgekehrt die Herauseinzelung eines autonomen Werks aus einem größeren Werk

36 Coulembier: Multi-temporality, S. 161.

37 Ebd., S. 170. 
und damit die Verungleichzeitigung eines Werks. In beiden Fällen bleibt der musikalische Text jeweils unverändert. Nicht eine bewusste äußere Maßgabe, sondern gerade die unabhängige Realisierung dieser Bedingungen durch die verschiedenen Komponisten bestätigt und festigt diese als Voraussetzung für die Entstehung des Poly-Werks als eine neue Gattung.

Beispiele für Poly-Werke in chronologischer Reihenfolge

$1948 / 49$

1975

1977

1973-1985

1982

$1982-2020$

$1985 / 86$

$1989 / 90$

1989/1992-1993

1990-1992

1994/95

1995-1996

1992-1997

1996-1998

$1997-2001$

1996-2011

1999

2001

2003

2003/05

2005/06
Darius Milhaud: Streichquartette Nr. 14 \& 15

Klaus Huber: Schattenblätter für Bassklarinette, Cello und Klavier

Klaus Huber: Oiseaux d'argent für drei Flöten

Vinko Globokar: Laboratorium für diverse Instrumente

Robert HP Platz: Flötenstücke für Altflöte und Kammerensemble

Julio Estrada: Yuunohui Cycle für diverse Instrumente

Klaus Huber: Protuberanzen für Orchester

Adriana Hölszky: Hängebrücken für zwei Streichquartette

Robert HP Platz: Grenzgänge STEINE für Sopran, zwei Klaviere und Orchester

Claus-Steffen Mahnkopf: Medusa für Oboe und Orchester

Georg Friedrich Haas: „... Einklang freier Wesen ... “ für Ensemble

Claus-Steffen Mahnkopf: Kammerzyklus für Klavier und Ensemble

Richard Barrett: Opening of the Mouth für Ensemble

Robert HP Platz: up down strange charm für diverse Instrumente

Bernd Franke: half-way house - Solo xfach für diverse Instrumente

Juliane Klein: Aus der Wand die Rinne für diverse Instrumente

Mark Randall Osborn: The Fluid Pronoun für Piccolo/Flöte, Ensemble und Tape

Bernd Franke: CUT I-III für Ensemble

Aaron Cassidy: the green is either für drei Trios

Simon Steen Andersen: Next To Beside Besides für diverse Instrumente

Robert HP Platz: Horizont Architektur Kern für Violine, Orchester, Tonband 
2007 Robert HP Platz: Unter Segel Boutaden Hülle 2 für diverse Instrumente

2008 Marko Ciciliani:Jeanne of the Dark für Ensemble, Licht und Video 2008 Chaya Czernowin: Anea Crystal Cycle für zwei Streichquartette

2008/10 Robert HP Platz: Wunderblock für Ensemble

2009 - $\quad$ Robert HP Platz: Branenwelten 1-6 für diverse Instrumente

2012 Valerio Sannicandro: Windströme und Seelenströme für zwei Orchester

2015/16 Robert HP Platz: dur moll hören für diverse Instrumente und Elektronik

2016/17 Ulrich Kreppein: Echoräume für vier Ensembles

$2017 \quad$ Karin Wetzel: X-Pieces für zwei Blockflöten

2017/18 Georg Friedrich Haas: Blumenwiese

2017/18 Valerio Sannicandro: Tactile Songs I und II für zwei Quartette

2019/20 Karin Wetzel: Dividuum für Violine 